\title{
VIRTUAL REALITY TO ALLOW WHEELCHAIR USERS TOURING COMPLEX ARCHAEOLOGICAL SITES IN A REALISTIC MANNER. TOWARDS THEIR ACTUAL SOCIAL INTEGRATION.
}

\author{
E. Pérez ${ }^{1}$, M.J. Merchán ${ }^{1}$, S. Salamanca ${ }^{1}$, P. Merchán ${ }^{1}$ \\ ${ }^{1}$ Escuela de Ingenierías Industriales. Universidad de Extremadura. Avda. de Elvas s/n. Badajoz. Spain. \\ \{emilianoph, mjmerchan, ssalaman, pmerchan\}@unex.es
}

\section{Commission II}

KEY WORDS: Virtual Reality, Digital Cultural Heritage, Disabled People, Motion Platform, Haptic Simulator.

\begin{abstract}
:
Technology development really reaches its highest point when it becomes a tool to facilitate life to people, especially to those more disadvantaged groups. This is the case of people with disabilities, who find lots of barriers to cope with the most usual and simplest things in their day-to-day. This fact is even more significant when they happen to face heritage buildings or environments. For instance, blind people or people with reduced mobility too often come upon architectural barriers that stop them from enjoying sites and monuments. Focused on people that have to use wheelchairs, a VR experience have been developed in order to provide them with the most realistic sensations while virtually touring on their wheelchairs some archaeological sites. This paper presents the steps forder the users to have the same sensations as if they were wheeling on the floor while visiting the site: irregularities, slopes and narrowness. The VR application has been developed to traverse one of the most monumental buildings of the whole Spanish Protohistory, the site of Cancho Roano (Zalamea de la Serena, Spain). The procedure to generate its digital 3D model from real data acquired with a laser scanner is also dealt with in it.
\end{abstract}

\section{INTRODUCTION}

One of the challenges the European Union proposes for the nearest future is to achieve the real integration of those sociocultural groups that are still not totally integrated. Cultural Heritage is currently perceived as a perfect tool for promoting social integration and that is why EU fosters all the initiatives that help to improve and promote cultural heritage experiences while providing disciplines such as Computing or Humanities with new research tools.

Following this course of action, people with disabilities must be considered a target since they are one of those socio-cultural groups not still sufficiently incorporated into enjoying such experiences. For instance, visually disabled people and wheelchair users, inside this very group, too often come upon architectural barriers when facing Cultural Heritage that stop them from appreciating sites and monuments.

Focused on people with reduced mobility, different technologies have been included to the field of rehabilitation engineering recently. The premise is to develop assistance systems for wheelchairs users that are adapted to their needs and can improve their quality of life. For this reason, the development of methods to evaluate motor and cognitive abilities that facilitate the process of choosing the appropriate technical assistance systems has been pursued for years. Applying these advances to facilitate a proper experience with Cultural Heritage is the next goal to reach. The creation of virtual visits to sites and monuments that implement the tools provided by rehabilitation engineering is one of the lines to follow in this sense. On the other hand, maybe this VR experiences could help to design more suitable infrastructures that can facilitate the real visit to this kind of environments.

In this perspective, an initiative like the one presented in this paper makes sense. It presents the 3D model of the pre Roman site of Cancho Roano (Zalamea de la Serena, Spain) and the VR application designed to visit it on a wheelchair.
Cancho Roano is one of the most monumental buildings of the whole Spanish Prehistory. Its lifespan covers from the end of the VII century - beginning of the VI century BC, at the height of the Tartessian culture, to the IV century BC, when the monument was intentionally destroyed by their users with a great fire. Several constructive phases have been documented during its lifetime, always preserving its religious significance. The site is located in the countryside in a hidden position near several streams, which made possible the inhabitants of the territory had contacts even with the Mediterranean cultures (Celestino, 2011-2012).

Nowadays, this site must be reached by car that has to be parked in the nearby. A brick path leads to the interpretation centre and then to the ancient building, which can be freely visited. To get to the monument, it is necessary to ascend a wooden ramp and climb one step to access to an open area. The main building that can currently be visited has a square plant of $24 \mathrm{~m}$ side, approximately. It consists of eleven rooms in different heights, some of them inaccessible to a common visitor due to preservation reasons, and a large patio with a characteristic Ushape. The entire building is surrounded by a slightly sloping terrace $2 \mathrm{~m}$ width, from which it is easy to see all the rooms in spite of the difficult walk to get there (Celestino, 2001) (Fig. 1). If this complex architecture hinders the visit in a regular tour, the more for wheelchairs users, for whom it is almost impossible to reach to the site main access and it is even worse if trying to go all over it due to the barriers imposed by slopes, narrow corridors, irregular floors, steps ...

In a case like this, it is evident that some tools to facilitate the visit of such a monument by disabled people must be designed. And here is where this proposal started. Following the procedure explained in section 3 , a VR experience combined with motion capture and motion simulation was created to provide a realistic tour.

After this statement of intents, the rest of the article is structured as follows. Section 2 describes the related works that can be found in the literature. Section 3 is dedicated to drawing the 
procedure for the 3D model creation, to defining the integration of all the devices composing the VR system that produce the VR application. The conclusions of this work are summarized in section 4.

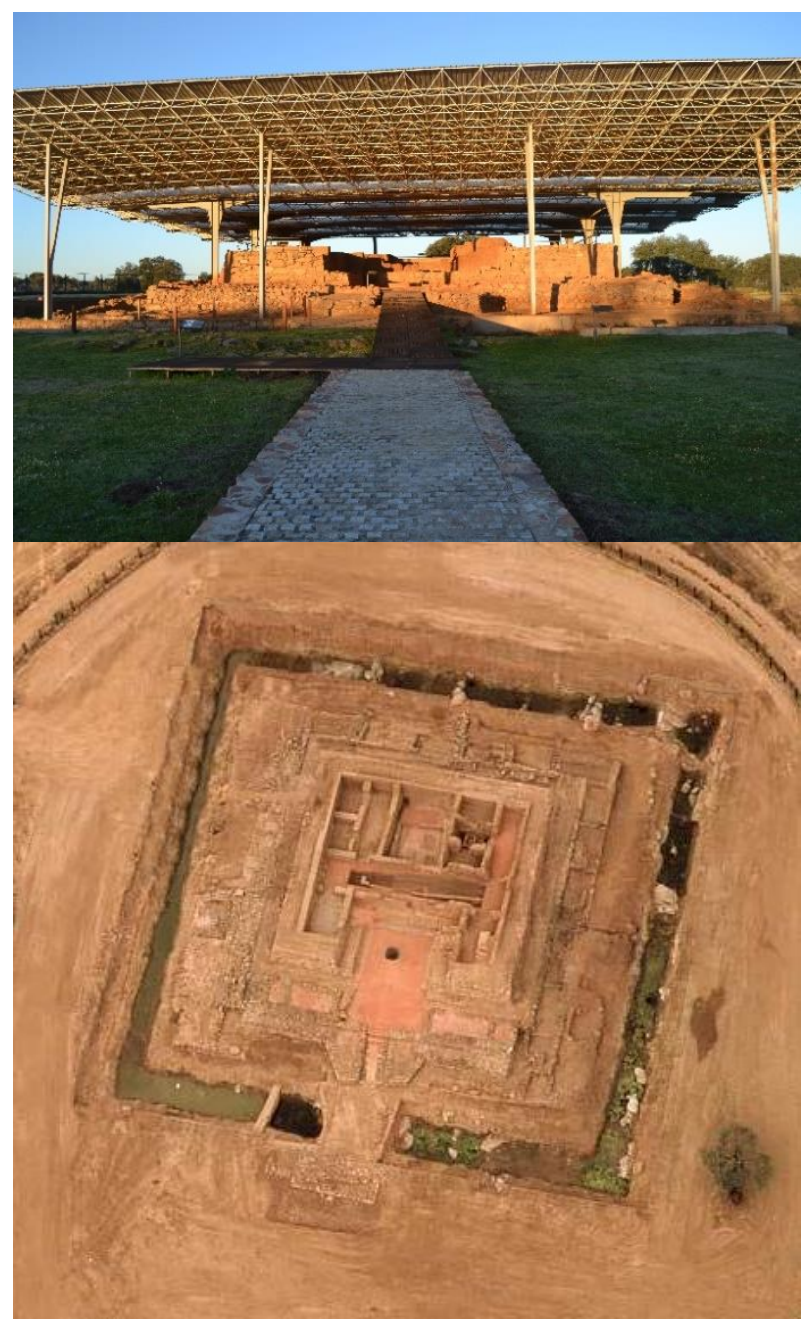

Figure 1: Cancho Roano. Above: Access and front view. Below: aerial view of the site.

\section{RELATED WORKS}

As said, different technologies have been incorporated to the field of rehabilitation engineering so far. Thus, together with experts in Neurosciences and Physical Medicine, several research groups have been working for the last years on prototypes of wheelchair simulators for users to ameliorate their day-to-day life by the creation of experiences in a risk-free environment (see, for example, the following revisions: Pithon et al. 2009 and Abellard et al. 2010). In this sense, the creation and validation of virtual environments have become an indispensable step so that the wheelchair users can simulate real movements and situations in order to improve the process, the sensations as well as the development of systems and devices (Mahajan et al., 2013; John et al., 2018).

From the very first task as learning the use of power wheelchairs to the training in the daily actions (working at the office, using a lift, etc.) there is a lot of literature that collects the state of the art, exposes the problematic to face and explains the different solutions reached (Abellard et al., 2010; Fernández-Panadero et al. 2012; Fernández-Panadero et al., 2014; Alshaer et al., 2017; Pinheiro et al., 2016; Tao \& Archambault, 2016; Devigne et al., 2017; Gallien et al., 2017).
With the conviction that applying the methodology developed in other contexts can also help to the real integration of people in wheelchairs in the society, its application to Cultural Heritage has been explored in this work. Mainly because, as mentioned, when visiting sites and ancient monuments, people with disabilities have to face architectural barriers that hinder their tour most of the time. With any luck, they can find narrow entrances and difficulties in access. But, unfortunately, many times they cannot tour the sites because it is dangerous or even, impossible in the absence of the proper facilities.

Virtual Reality is being increasingly used in Cultural Heritage (Christofi et al., 2018; Drossis et al. 2018; Pérez et al., 2018), although it has been utilized mostly for educational (see, for instance, Froschauer et al., 2013 or Mortara et al., 2014) and dissemination purposes (Valtolina et al. 2005, like one of the first examples). Addressing the issue of impossibility of going to certain sites or museums, some virtual visits and tours to be made from the computer appeared, and a huge number of webpages can be visited nowadays. Wheelchairs users are indirect beneficiaries of these initiatives. But the cases on which VR has been applied to provide vivid experiences for wheelchairs users as if they were really wheeling on monuments or sites are scarce, not to say non-existent. Even rarer are the occasions in which these VR applications have been made to tour the models created from the actual data of the cultural heritage space.

\section{DEVELOPED APPLICATION}

The main goal of this VR experience is to let the user explore the archaeological sites from an accessible position with the same realistic sensations as if he/she was promenading the actual place on his wheelchair.

Essentially, the tasks leading to obtain the VR application can be classified into two main phases: the generation of the 3D model of the site to be virtually traversed and the design of the application with its corresponding interface. These two phases are described in the subsections below. A simple scheme of the procedure is shown in Figure 2.

Morover, the other important task to reach our proposal is the integration of several technologies composing our VR system, that is, a VR simulator with motion capture and motion simulation.

\subsection{D model generation}

To generate the 3D model of Cancho Roano, a Faro LS 880 laser scanner that provides panoramic views of the scene was used to acquire the geometry. For this device, azimuth and elevation angles range over $\left[0,360^{\circ}\right]$ and $\left[-65^{\circ}, 90^{\circ}\right]$, respectively, and the maximum data density is $40.110 \times 17.223$ points. Obviously, when used in outdoor spaces most of the potential data are useless because they correspond to the sky. A Nikon D200 camera with Nikon AF DX Fisheye lens coupled to the scanner provides the colour information. It composes a single panoramic image from 10 photos, 10.2 MPixels each. The system takes about 27 minutes to scan a panoramic sample (at half the maximun resolution) plus 8 minutes to take the sequence of photos. Data acquisition was made during 3 sessions, 10 hours per session.

It is beyond dispute that the first task to be accomplished once in situ is the planning of the accessible scanner positions. The volume that could be covered in a single scan is affected by several factors, namely, the field of view, the occlusion conditions, the accessibility of the scanner, and the overlap with other scans. 


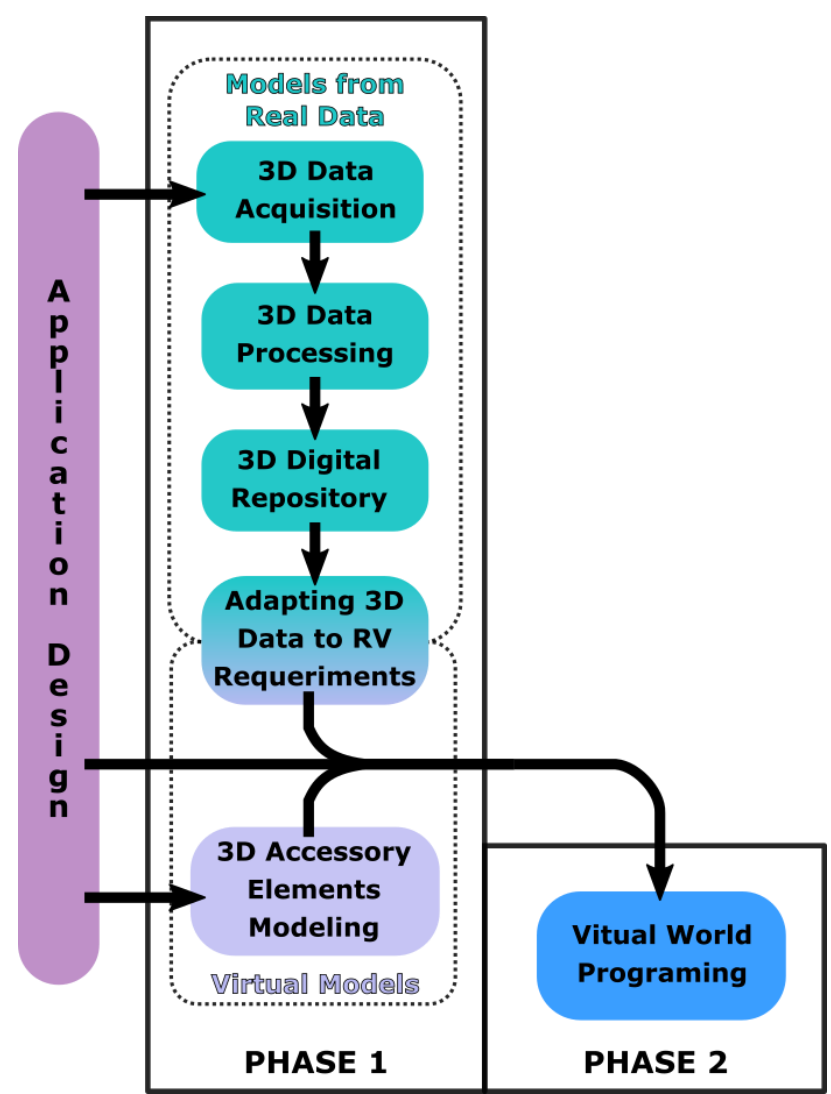

Figure 2: Scheme of the procedure followed to build the VR application.

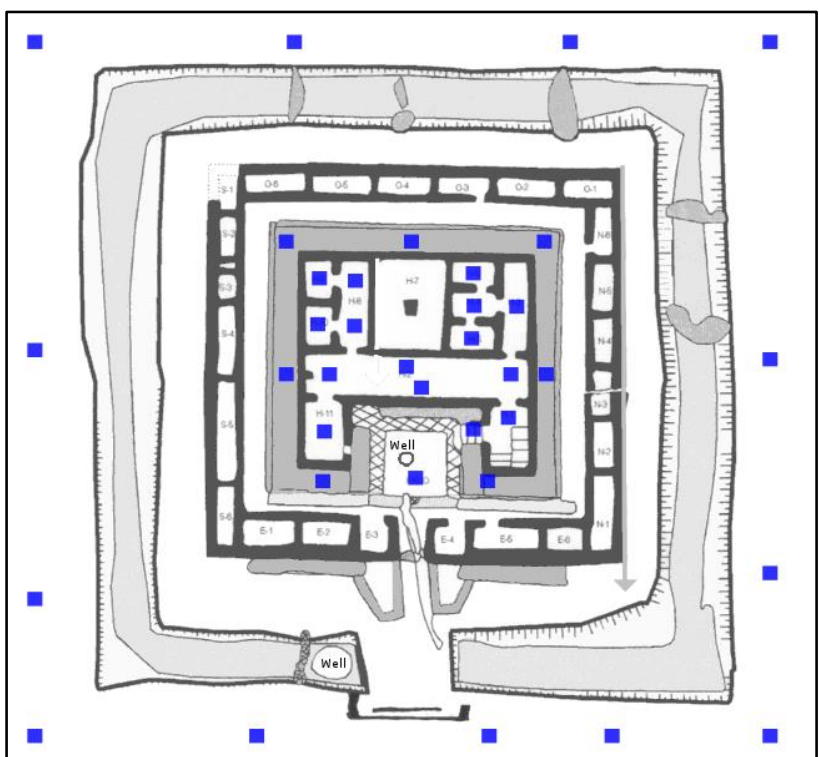

Figure 3: Blue spots point out the scanners positions on a map of the site.

In the case of this Heritage Building, the scans were taken in a series of concentric rings. Figure 3 shows the 33 scanner positions indicated on a map of the site.

Before beginning the generation of the model a noise and outlier filtering pre-processing task is carried out. This filtering process eliminate erroneous points provided by the scanner: points generated in the infinite (spread points), noise (owing to reflections and shiny surfaces) and outliers (points outside the scene). After that, the acquired partial views have to be registered. Registration signifies the process of putting in correspondence two overlapped point clouds so that they are in the same coordinate system. Data registration is a well-studied problem, and methods to automatically register laser scans data are commercially available. After this process, a coarse transformation is achieved. The registration refinement stage calculates an accurate alignment between both point clouds Finally, the merging stage samples the points and rewraps it in order to make smooth transitions and generate only one cloud of points.

Apart from noise and outliers filtering tasks in the preprocessing stage, we had to correct errors in colour assignment. The system is capable of associating colour information to each point of the scene but, due to small camera alignment errors, the colour-geometry registration must be refined. To solve this problem, we set a pair of corresponding points in reflectance and colour images and aligned accurately.

\subsection{Creation of the virtual world}

The procedure of the creation of the 3D model explained before is aimed to obtain an accurate digital representation of the Heritage place. As a result, a high-resolution colored 3D model is obtained that could be used in several applications: replica creations, meaningful measurements of constitutive elements, high quality rendered images from any desired point view, etc. Our targeted application is the use of this data to be represented in real time so as the user can virtually visit the ruins.

Representation in real time and high-resolution models are concept that are not compatible nowadays, due to the constraints imposed by the current hardware. Therefore, one essential first step to reach virtual visits is to apply some adaptations to the digitized and processed 3D data. Moreover, since the data obtained after the model generation stage consist of a colored point cloud and the virtual application engine (Unity) requires triangulated meshes, a conversion of type of data is necessary. Finally, it is also important to adapt the color information format of the $3 \mathrm{D}$ model, converting it into a useful format accepted by Unity.

Therefore, the adaptation process can be divided into three steps:

- Resolution reduction: the number of points of the model must be reduced in order to obtain a model that could be represented in real time in an average current computer. This reduction is carried out with the use of the 3D open source processing software Meshlab, which is well-known and very spread among the Computer Vision Scientific community. Specifically, we applied the simplification "Quadric Edge Collapse Decimation" to the original point cloud of $15,000,000$ points, resulting in a reduction of $30 \%$ data, that is, a point cloud with $4,500,000$ points.

- Type of data: the point cloud has to be converted into a triangulated surface. First, all the normal in the point cloud are oriented by using the tool "Compute normal" of Meshlab software. Then, a reconstruction process using the "Screened Poisson method" is applied. The final triangulated surface is composed by $8,000,000$ triangles.

- Color conversion: this is carried out by means of the Meshlab tools named "Parametrization Trivial per-triangle" (to map all triangles into equal sized triangles) and "Transfer Vertex attributes to texture" (to assign a color per vertex to the mesh's texture), obtaining a JPG image file that stores the color information.

On the other hand, non-adapted buildings present architectural barriers to be travelled by disabled people in wheelchairs. The archaeological ruins can be considered an extreme case of nonadapted building. Given that the use of the simulator is intended to make the user feel as if it were actually in the historic site 
using his/her wheelchair, the need to design certain technical aids, such as ramps, floors and lifts, is imposed. Thus, he/she can make the visit in similar conditions to those of a person without a wheelchair. These aids could even be designed so that the user can get into the inaccessible rooms, so they can enjoy being inside, improving their feelings in comparison with a common visitor.

Consequently, a controllable interface has been designed in the application in which the user can activate and control some technical aids in the virtual world: ramps and elevators, using a controller attached to the wheelchair (Figure 4 (b) and (c)).

The 3D models of these accessories were designed from scratch taking into account all the considerations of real time and Unity requirements. Therefore, it is not necessary to apply an adaptation to such elements.

\subsection{The simulator}

We have developed a motion simulator with haptic interface to let the disabled people experience the movement sensations while they tour a Heritage place (Figure 4 (a)). Besides, they can decide the trajectory by moving manually the wheelchair wheels as if they were moving across the real place. This simulator follows the schema shown in Figure 5. It is integrated by the following devices:

- A workstation that is the main component of the simulator, essential for its operation since it makes possible the communication between all the elements. The application for the management of the simulator has been developed in Unity, software that allows to easily incorporate models of virtual worlds and interact with them. Also, this software allows the management of any hardware device that has the corresponding programming libraries, thanks to the possibility of introducing scripts in $\mathrm{C \#}$ language. It is important to note that the graphics power of this PC limits the level of detail or the complexity of the virtual worlds that are designed. In addition, if you want to simultaneously manage several viewing outputs (monitor, 3D screen and head mounted display, HMD) it is recommended that the graphics card installed has that functionality. In this simulator two graphics cards are used simultaneously: Nvidia GTX970 and Nvidia Quadro K5200.

- An interface with the wheelchair, consisting of a moving platform, another haptic for the chair and access elements: It is constituted by a moving platform of six degrees of freedom and a haptic system. The platform allows the user to transmit the accelerations to which he/she is subjected as an effect of the movements that take place in the virtual world. It also allows reproducing the different inclinations of the terrain of the virtual world on which the user is moving. Concerning the haptic system, it consists of two active cylinders driven by motors that serve as the interface with the wheelchair, which must be positioned so that the wheels are in permanent contact with the cylinders. They have a double function: on the one hand, the cylinders are activated when the user moves the wheels and detect the intention of the user's movement. And, on the other, they emulate the conditions of the surface of the virtual world with the movement of the rollers. That is, depending on the inclination and type of surface of the virtual world in which it is located, the user may even notice more or less ease when it comes to moving the wheels. The motion platform and the active cylinders mounted can be observed in Figure 6. The workstation is responsible for measuring and processing the movement of the rollers, as well as generating the rotation to the rollers that corresponds to the one experienced by the wheels of the virtual wheelchair. The communication between the workstation and both platforms is done through a router with a Gigabit Ethernet connection, using the TCP protocol. The interaction between the virtual world and both platforms is managed from a script in Unity;

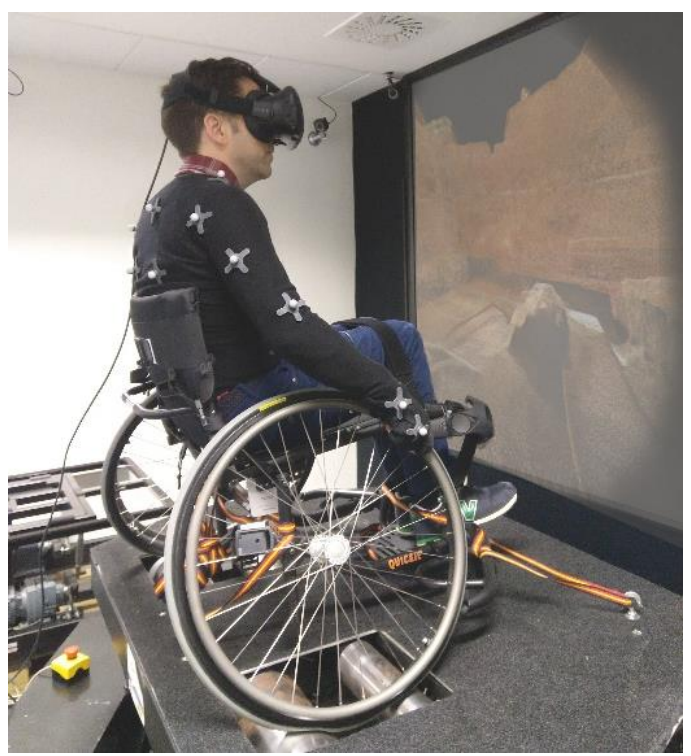

(a)

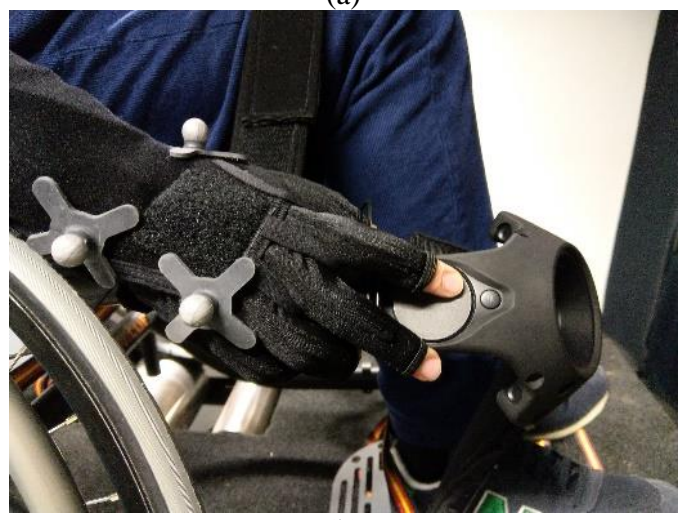

(b)

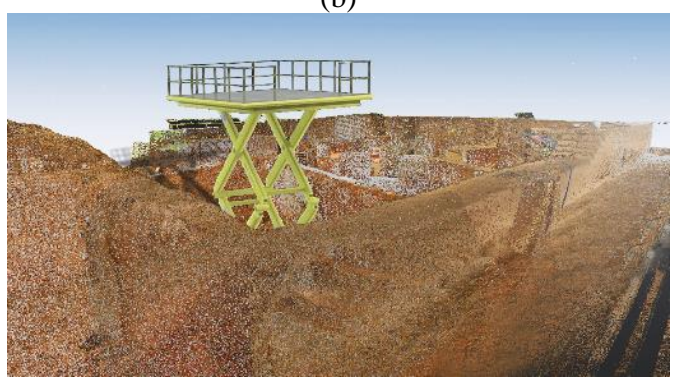

(c)

Figure 4: Resulting virtual application with the motion simulator: (a) User exploring the Cancho Roano ruins with the motion capture suite, data gloves and haptic motion system; (b) controller attached to the wheelchair to control the user interface; (c) elevator inserted within the ruins as technical assistance to visit inaccessible zones. 


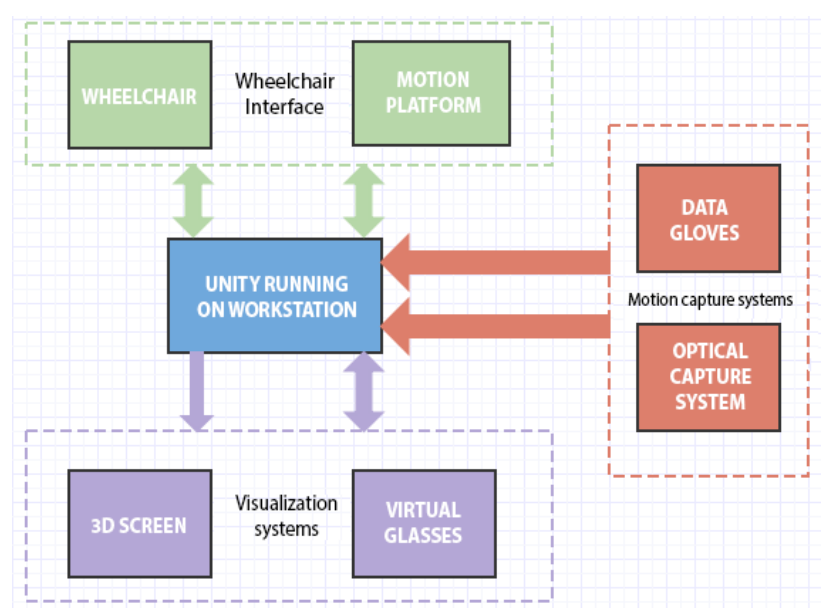

Figure 5: Scheme of the VR system composed of different types of devices.

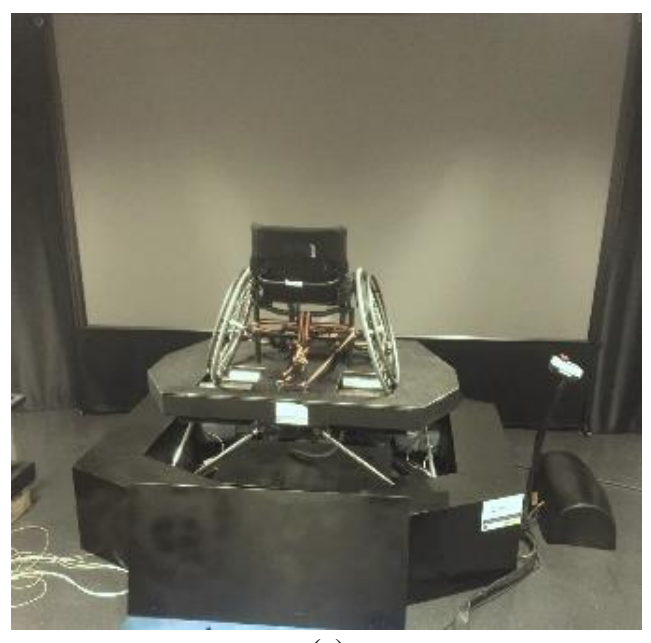

(a)

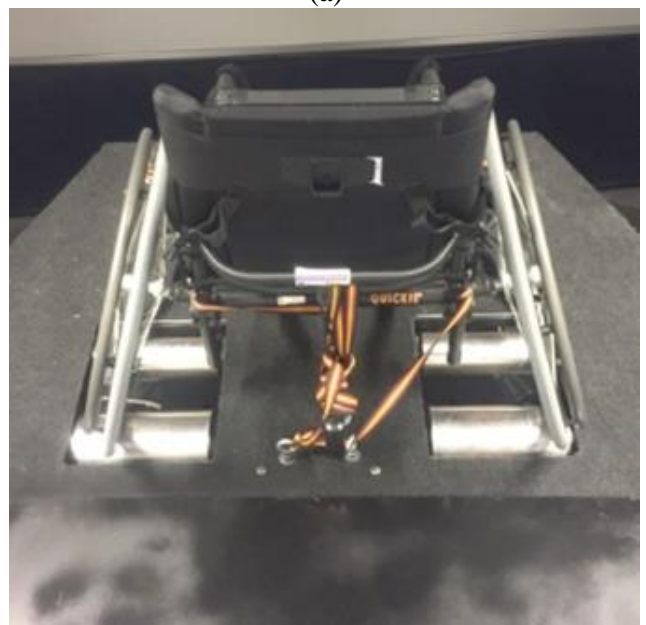

(b)

Figure 6: Interface with the wheelchair: a) 6DOF motion platform; b) active cylinders mounted on the motion platform.

- $\quad$ Capture system: to capture the movements of the user (see Figure 7), the simulator has an optical system, to obtain the measurements of the movement of the entire body (OptiTrack), and a 5DT data glove to record the movement of the fingers. This system has a double function: 1) register data for further processing, analysis and study in order to diagnose possible problems or erroneous behavior in the user; and 2) improve the feeling of immersion of the user, since he/she sees that his avatar moves synchronized with the movement of his body. The software for the control of the OptiTrack capture system is Motive. This software allows the calibration and configuration of the system and provides interfaces that in turn allow the capture and processing of $3 \mathrm{D}$ data. In addition, it also allows a broadcast (streaming) of the data captured through the network, which offers, in turn, the possibility of transferring them to the avatar of the virtual world through Unity and C \# Script.

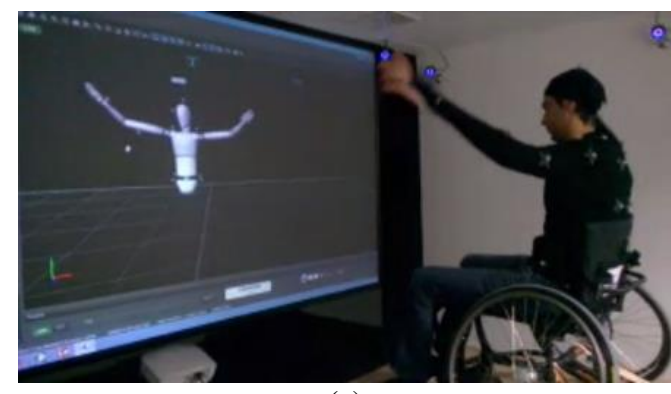

(a)

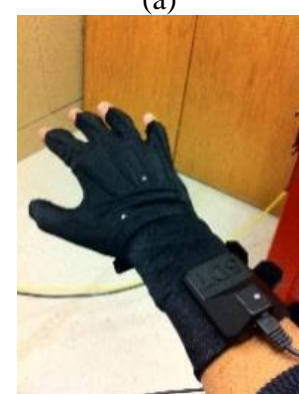

(b)

Figure 7: Motion capture systems: a) optical capture system; b) data gloves.

- Visualization devices: Regarding the visualization of the virtual world, the simulator has a large $3 \mathrm{D}$ screen and a HMD: HTC Vive. The user of the wheelchair uses the HMD so that, thanks to immersion, he/she can perform his movement routines as he/she usually does in the real world. On the other hand, the 3D screen allows the technical team to observe at all times how the user is acting and, in this way, to give the pertinent orders.

\subsection{Integration of devices in the VR system}

This section describes the processes of integration of the workstation with the capture system, the moving platform/haptic system and the HMD through Unity in order to create the virtual worlds for the simulator

The integration of OptiTrack optical body capture system with Unity was done using the plugin provided by the manufacturer following these steps:

1. Definition of a skeleton in Motive, exploiting one provided by the software (full body of 27 markers).

2. Activation of streaming data transmission from Motive through Multicast protocol.

3. Loading the plugin in Unity and assigning the script to a Unity skeleton that must have a certain hierarchical structure.

4. Linking between the Unity skeleton and the Motive skeleton (the same name must be assigned to both of them). 
A fundamental step for the connection between both programs is to study the relations between the coordinates systems that each of them uses and to establish a process of adaptation between both of them. The capture software, given the physical distribution of the camera system, fixes the origin of the reference system at the centre of the capture volume, $\Sigma_{\text {capture }}$ (Figure 8 (a)). This point corresponds to the initial position of

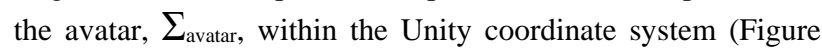
8). Our application must achieve that, once the simulation is launched, if the user is in the middle of the room, at any point of the capture volume of Optitrack, when he/she physically moves towards the real wheelchair and seats, the virtual avatar that appears in Unity must also approach the virtual chair and seat in it. This requires two fundamental steps:

1. Change the origin of coordinates of the capture volume in Motive, placing it in the centre of gravity of the chair. To do this, the distance between the centre of gravity of the cameras, capture, and the point of the actual wheelchair that coincides with the point where the centre of gravity of the virtual wheelchair is located in Unity must be measured, $\Sigma_{\mathrm{w}}$. This distance is applied as an offset to Motive's reference system (Figure 8 (a)) so that the new reference system will be located where it is shown in Figure 8 (b).

2. As can be seen in Figure 9 (a), in Unity, initially, the avatar and the wheelchair have their respective centres of gravity, $\Sigma_{\text {avatar }}$ and $\Sigma_{\mathrm{w}}$, with respect to the

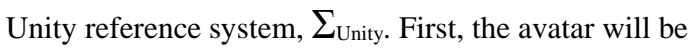
made dependent on the wheelchair, so that its absolute reference system is $\Sigma_{\mathrm{w}}$ (Figure 9 (b)). Next, the avatar will be moved to its new origin of coordinates, resulting in $\Sigma_{\text {avatar }}$ and $\Sigma_{\mathrm{w}}$ being coincident (Figure 9 (c)).

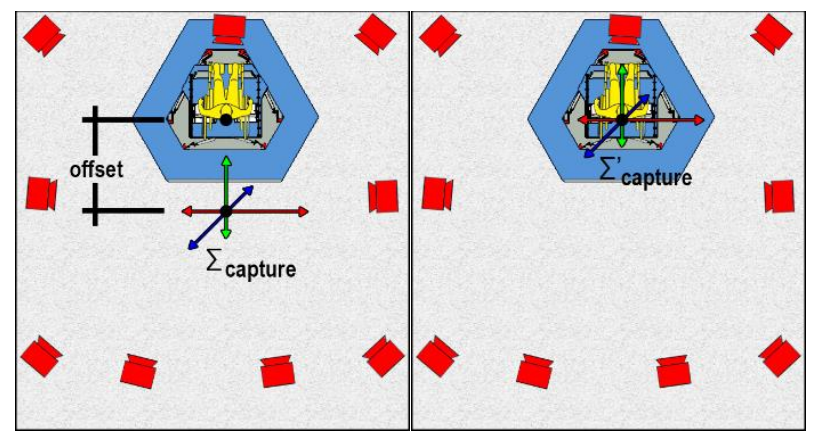

Figure 8: Displacement of the reference system in the capture software Motive: (a) Initial reference system, $\Sigma$ capture, and distance between $\Sigma$ capture and the centre of gravity of the wheelchair (Offset) (b) New reference system for the capture software, $\Sigma$ 'capture (In red, the 9 cameras of the capture system are represented, in blue, the movement platform and, in yellow, the user on the wheelchair).

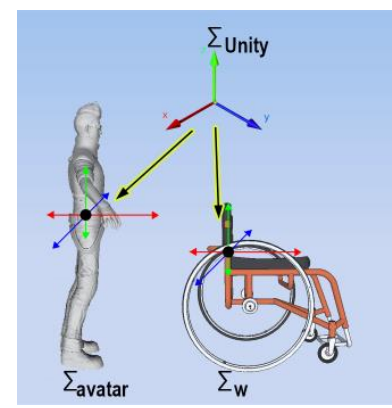

(a)

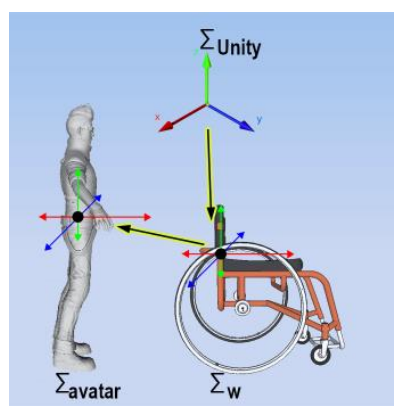

(b)

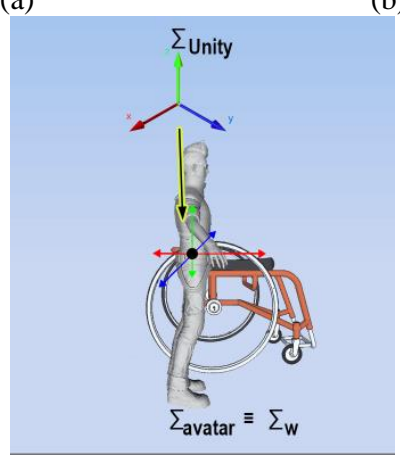

(c)

Figure 9: Assignment of reference systems in Unity: (a)

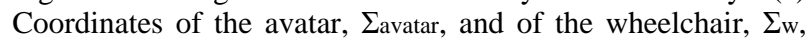
located in their centres of gravity, with respect to the Unity reference system, ¿Unity (b) Assignment of the centre of gravity of the chair as the avatar's main reference system (c) Displacement of the avatar to its new origin of coordinates, $\Sigma_{\mathrm{w}}$

In order to integrate the platform and the wheelchair in the Unity environment, it is necessary to model both of them inside the software.

Unity incorporates a physical engine, based on PhysX, which analyses in real time the physical interactions between the elements due to the contact between them and the gravitational force. To control the platform from Unity, a script that allows the bidirectional communication has been developed, according to the scheme of Figure 10, with two main functions:

1. Detecting the movements made by the chair in Unity, as well as the accelerations that the virtual model experiences and transmit them to the platform.

2. Capturing the orders of movement that the user gives, that is to say, the angle and the speed of rotation of each one of the wheels, through the roller system of the platform. These commands are automatically transferred to the Unity visual representation which has an effect directly on the world that the Unity physics engine is processing.

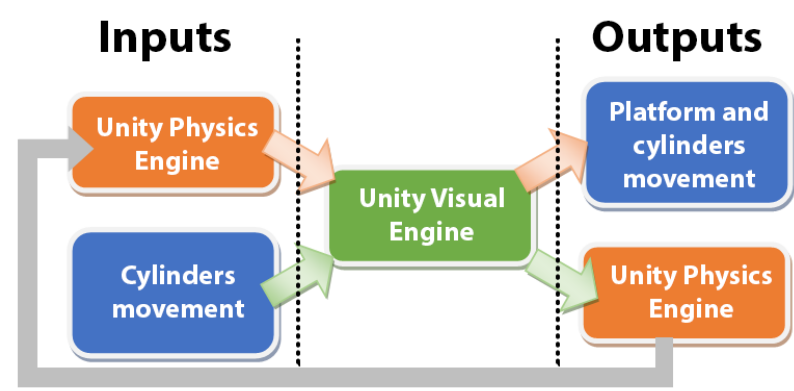

Figure 10: Diagram of bidirectional communication between the movement platform and Unity. 
The developed script allows the configuration of a series of parameters, both the platform and the virtual world, among which include:

- 'Platform Config File': external XML file in which the configuration parameters of the platform are set, such as the speed of the motors.

- $\quad$ 'Right / Forward / Up Linear or Angular Scale ': a linear or angular scale of the right axis, front or top, respectively.

- 'Factor 1 ': factor by which the speed of the chair is multiplied when moving along a slope. Positive values simulate a resistance to low movement, while negative values, high.

- $\quad$ 'Factor 2 ': factor by which the speed transmitted from the two rollers to the virtual chair is multiplied. Note that by selecting negative values of this factor, it is possible to reverse the direction of movement, whereas if a null value is chosen, the virtual chair does not move.

- 'Factor 3 ': a multiplier of the speed of the rollers. For negative values, this factor can be used as a friction factor of the chair on the surface on which it is moving. On the contrary, if a positive value is chosen, it can be used as a negative friction, as it could be in the case of an icy surface.

It should be noted that a method has been created to configure the interaction between the virtual world and the roller system, by means of which different values are assigned to factors 1,2 and 3 to characterize different types of surfaces in the virtual world, such as concrete, grass, sand and neutral, which basically differ by having different coefficients of friction. This method makes it possible for the behaviour of the rollers to emulate the friction of the chair with the specific surface when the wheelchair passes through each type of floor. For the moment, the adjustment of these parameters has been done empirically and with illustrative values of soils with large differences in their coefficients of friction in order to be able to perceive the differences in behaviour of the haptic system.

With respect to the synchronization between the virtual movements and the real ones of the platform, no delays are observed in the response times, even selecting a speed of the engines below the maximum in 'Platform Config File'. Obviously, with respect to the movements of the platform, it simulates those of the virtual wheelchair with the limitation imposed by the dimensions and relations between the mechanical elements that form the motion platform.

For the representation of the wheelchair in Unity, it is necessary to take into account two issues. On the one hand, the 3D model of the wheelchair with its corresponding properties (dimensions, materials, the centre of gravity, material contact with the ground), which will have their effect within the Unity physics engine (Figure 11). As can be seen, the model is basically divided into a chassis and the two main driving wheels. Each of these elements must be associated with the property called Collider, which causes the object to be taken into account within the physical engine. In particular, each collider has to be given a shape and dimensions, that is, the collider defines the envelope of the 3D model to which it is associated, from which the calculations for the collisions between objects will be made. A cubic collider has been defined for the chassis and two specific colliders for Unity for the wheels.

For the HMD, the integration in Unity has been carried out by means of the plugin VIVE Input Utility provided by HTC. Although this plugin perfectly solves the integration of the HMD in Unity, it is worth mentioning that for the absolute positioning of the HMD, not to use the external camera has been decided but the optical capture system. Specifically, 6 optical markers have been attached to the HMD, distributed asymmetrically. This distribution was chosen in order to be able to differentiate perfectly where the user is looking at. The steps followed for the integration of the hull in Unity are as follows:

1. Loading the HTC plugin in the Unity scene.

2. Assignment of the HMD controller script to the head of the avatar.

3. Deactivation of the absolute positioning functionality.

4. Creation in Motive of a rigid solid defined by the 6 markers attached to the HMD.

5. Activation of streaming data transmission from Motive.

6. Loading the Optitrack plugin in Unity and assign the rigid solid script to the head of the avatar.

7. Connection of the solid of Unity with the solid of Motive (they must be assigned the same name).

8. Monitoring checking and visualization with the HMD.

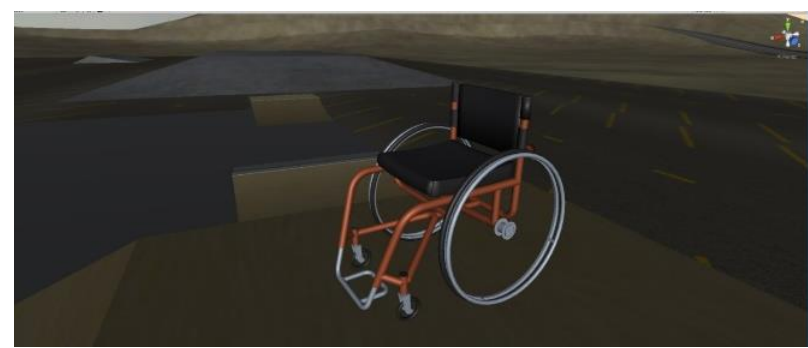

Figure 11: 3D model of the wheelchair used in the application developed in this work.

\section{CONCLUSIONS}

This work presents a VR application that allows people in wheelchairs to visit the archaeological site of Cancho Roano (Zalamea de la Serena, Badajoz), one of the most monumental buildings of the whole Spanish Prehistory.

This application permits users to know a place of high historical value but, especially, it helps people with disabilities attain a real social integration that has not yet been fully achieved in the field of dissemination of cultural heritage. In order to reinforce this aspect, two strategies have been used in addition to the classic VR procedures. The first one is to make the tour experience through the site as realistic as possible for a visitor in a wheelchair. The second one to create the 3D models from 3D data acquired with laser scanners, which allows a visualization of the site as it is currently in reality.

To make the tour as realistic as possible, a moving platform with a haptic roller system, a motion capture system and a virtual reality helmet have been used and a simple basic application has been developed for the simultaneous use of these technologies. In this application, different types of soil have been empirically modelled that provide realism to the user's experience, while he/she is travelling with the wheelchair on these floors virtually.

To create the $3 \mathrm{D}$ models, the models generated from the $3 \mathrm{D}$ data acquired with a Faro LS-880 laser scanner have been conveniently adapted to be integrated into the VR application.

\section{ACKNOWLEDGEMENTS}

This work has been supported by the project IB16162 from Junta de Extremadura and Fondo Europeo de Desarrollo Regional "Una manera de hacer Europa".

\section{REFERENCES}

Abellard, P., Randria, I., Abellard, A., Ben Khelifa, M.M. and Ramanantsizehena, P., 2010. Electric Wheelchair Navigation Simulators: why, when, how? A. M. Donato Di Paola and G. Cicirelli (Ed.), Mechatronic Systems Applications, InTech. 
Alshaer, A., Regenbrecht, H. and O'Hare, D., 2017. Immersion factors affecting perception and behaviour in a virtual reality power wheelchair simulator. Applied Ergonomics, Vol. 58, pp. $1-12$.

Celestino, S., 2001. Cancho Roano.

Celestino, S., 2011-2012. Arqueología Protohistórica de la Serena. CUPAUAM, 37-38, pp. 297-305.

Christofi, M., Kyrlitsias, Ch., Despina, M.G., Anastasiadou, Z., Michaelidou, M., Papamichael, I. and Pieri, K., 2018. A tour in the archaeological site of Choirokoitia using virtual reality: a learning performance and interest generation assessment. Advances in Digital Cultural Heritage. Lecture Notes in Computer Science, Vol. 10754, pp. 208-217.

Devigne, L., Babel, M., Nouviale, F., Narayanan, V., Pasteau, F. and Gallien, P., 2017. Design of an immersive simulator for assisted power wheelchair driving. IEEE Int. Conf. on Rehabilitation Robotics, ICORR'17, Jul 2017, UK.

Drossis, G., Birliraki, Ch. And Stephanidis, C., 2018. Interaction with Immersive Cultural Heritage Environments Using Virtual Reality Technologies. 20th International Conference, HCI International 2018. USA, pp. 177-183.

Fernández-Panadero, C., Cruz, V., Morán, D. and Delgado, C., 2012. PhyMEL-WS Weelchair Simulator: A Preliminary Study to Increase Awareness about the Problems of Living the City in a Wheelchair. Proc. 3rd European Immersive Education Summit, pp. 210-221, UK.

Fernández-Panadero, C., Cruz, V., Morán, D. and Delgado, C., 2014. PhyMEL-WS: Physically Experiencing the Virtual World. Insights into Mixed Reality and Flow State on Board a Wheelchair Simulator. Journal of Universal Computer Science, Vol. 20(12), pp. 1629-1648.

Froschauer, J., Merkl, D., Arends, M. and Goldfarb, D., 2013. Art History Concepts at Play with ThIATRO. Journal on Computing and Cultural Heritage, Vol 6, n 2, pp. 7- 15.

Gallien, P. et al., 2017. Virtual reality simulator for speeding up research on assisted power wheelchair navigation. Annals of Physical and Rehabilitation Medicine, Vol. 605, pp. 91-93.

John, N.W., Pop, S.R., Day, T.W., Ritsos, P.D. and Headleand, Ch.J., 2018. The Implementation and Validation of a Virtual Environment for Training Powered Wheelchair Manoeuvres. IEEE Transactions on Visualization and Computer Graphics, Vol. 24, Is. 5, pp. 1867-1878.

Mahajan, H.P., Dicianno, B.E., Cooper, R.A. and Ding, D., 2013. Assessment of wheelchair driving performance in a virtual reality-based simulator. J Spinal Cord Med., Vol. 36(4), pp. 322-332.

Mortara, M., Catalano, C.E., Bellotti, F., Fiuccy, G., HouriPanchetti, M. and Petridis, P., 2014. Learning cultural heritage by serious games, Journal of Cultural Heritage, Vol. 15, Is. 3, pp. 318-325.

Pérez, E., Merchán, M.J., Moreno, M.D., Merchán, S. and Salamanca, S., 2018. Touring the Forum Adiectum of Augusta Emerita in a virtual reality experience. Lecture Notes in Computer Science, Vol. 11196, pp. 548-559.

Pinheiro, O.R., Alves, L.R.G., Romero, M.F.M. and de Souza, J.R., 2016. Wheelchair simulator game for training people with severe disabilities. Proceedings of the 1 st International Conference on Technology and Innovation in Sports, Health and Wellbeing (TISHW).
Pithon, T., Weiss, T., Richir, S., Klinger, E., 2009. Wheelchair simulators: A review. Technology and Disability, Vol. 21(1), pp. 1-10.

Tao, G. and Archambault, P.S., 2016. Powered wheelchair simulator development: implementing combined navigationreaching tasks with a 3D hand motion controller. Journal of NeuroEngineering and Rehabilitation, 13:3.

Valtolina, S., Franzoni, S., Mazzoleni, P. and Bertino, E., 2005. Dissemination of Cultural Heritage Content through Virtual Reality and Multimedia Techniques: A Case Study. 11th International Multimedia Modelling Conference, Australia, pp. 214-221. 